\title{
Neutrophil Gelatinase-Associated Lipocalin as an Early Marker for the Diagnosis of Urinary Tract Infections in Saudi Children
}

Osama Y Safdar ${ }^{1}$, Mohammed A Shalaby ${ }^{1}$, Wael M Toffaha ${ }^{2}$, Alanoud A Turki², Waleed M Toffaha ${ }^{2}$, Afaf H Ghunaim², Sundus M NoorSaeed², Ahmad M Alghamdi ${ }^{2}$, Alaa K Bahashwan ${ }^{2}$, Malak S Alharbi², Ahmed M Balamash ${ }^{2}$, Mohammed F Almoflihi ${ }^{2}$, Khalid M Baghdadi ${ }^{2}$, Ghazi M Sindi², Asmaa K Baothman², Nouf F Aljahdali' ${ }^{2}$, Musab A Bukhari² and Jameela A Kari ${ }^{1}$

${ }^{1}$ Pediatric Department, Faculty of Medicine, King Abdulaziz University, Jeddah, Saudi Arabia

${ }^{2}$ College of Medicine, King Abdulaziz University, Jeddah, Saudi Arabia

\begin{abstract}
Background: Urinary tract infections (UTI) are widespread infections in the pediatric age group which presents non-specific symptoms. "Neutrophil gelatinase-associated lipocalin" (NGAL) is one of a biomarker in determining kidney injury. The early urinary tract infections diagnosis is essential for the purpose of preventing enduring consequences.
\end{abstract}

Aim: To evaluate the use of NGAL as an early predictor of UTI in clinical practice.

Patients and methods: A cross-sectional study examined specificity and sensitivity of urinary NGAL in the early UTI diagnosis. All febrile children admitted to KAAUH (age: 0-14 years) were included. Mann-Whitney U test, ShapiroWilk's test, and Spearman correlation was used to analyze the data.

Results: In terms of mean urinary NGAL, no significant difference was observed between urinary NGAL levels in patients with and without UTIs ( $P$-value $=0.17$ ). The receiver operator characteristic $(R O C)$ curve demonstrated 0.594 sensitivity, and 0.683 specificity. For serum CRP (ROC) curve demonstrated 0.53 sensitivity and 0.47 specificity. Spearman's correlation was undertaken to examine an association between NGAL and CRP levels. There was a positive monotonic correlation between NGAL and CRP levels $(r s=0.503 n=73, p<.001)$.

Conclusion: Urinary NGAL is a poor biomarker for the diagnosis of febrile UTI. Further investigations are required with larger groups of patient to confirm the results of this study.

\section{Keywords: NGAL; Urinary tract infection; Children}

\section{Introduction}

Urinary tract infections (UTIs) are one of the most widely studied infectious illnesses encountered by pediatric healthcare providers [1]. Pediatric patients with UTIs can present with non-specific symptoms such as fever, vomiting, and decreased oral intake. This leads to a delay in both the diagnosis of UTI and initiation of treatment, thus increasing the risk of complications [2]. The untimely diagnosis as well as treatment of UTI is essential. Delayed or ignored UTI diagnosis may cause malfunction in offering possible long-term consequences and suitable treatment, comprising of hypertension, renal scarring, as well as chronic renal failure [3]. The supplementary rigorous UTI forms can consequently result in acute complications such as dehydration and acute kidney injury (AKI).

In current clinical practice, UTI can be detected by using a variety of tests. These include screening tests, such as dipstick urinalysis from midstream urine that measures leukocyte esterase, nitrite, blood, and proteins. Diagnostic tests include positive microscopy and urine culture, if it demonstrates more than 100,000 colonies from a single organism and 10 white blood cells per high power field (WBCs/HPF) [2].

The culture of urine is considered to be a gold standard to diagnose UTI. However, positive results of culture needs two to three day for accomplishing identification of bacteria. Recently, the nitrite levels, leukocyte esterase, $\mathrm{pH}$ of urine are employed to screen for the condition of UTI, however, sensitivities for such test are about 70-80\% [2]. For the purpose of early UTI diagnosis, researchers are now exploring other biomarkers. Currently, various biomarkers are demonstrated for early AKI diagnosis comprising of kidney injury molecule-1 (KIM1), neutrophil gelatinase-associated lipocalin (NGAL), interleukin-18 (IL-18), and cystatin C, [4]. NGAL is one of an original protein identified in the granules of human neutrophils. It is also considered to be a constituent of the innate immune system. NGAL has a major role in several cellular responses, comprising of proliferation, bacteriostasis, differentiation, and apoptosis $[5,6]$. NGAL is a lipocalin, a small $25-\mathrm{kDa}$ extracellular protein. It is bounded covalently to the matrix metalloproteinase 9 that is specifically expressed by different neutrophils and displays antibacterial characteristic. Hence, this lipocalin is thought to be an innate immune system constituent [7]. NGAL is articulated at reduced levels in various tissues for instance, liver, kidney, and epithelium. It actively participates in the proliferation, development, and differentiation of quite a few human tissues. NGAL expression is induced in the epithelial cells by inflammation or by other injuries such as infection, intoxication, ischemia, AKI, and neoplastic transformation [7].

Current evidences have demonstrated that urinary and serum NGAL levels indicate a specific, sensitive, and novel biomarker for the untimely AKI detection as NGAL is stimulated quickly and released from the renal tubular cells with regards to insults on the kidney [8]. The measurements of NGAL are employed as early AKI biomarkers in a heterogeneous pediatric ICU setting and are capable to predict

*Corresponding author: Osama Y Safdar, Assistant Professor of Pediatrics, Department of Pediatrics, King Abdulaziz University Hospital, Alsulimania, Jeddah, PO BOX 14071, Postal code 214141, Saudi Arabia, Tel: +966505620849; E-mail: safderosama@hotmail.com

Received: September 21, 2014; Accepted: October 30, 2015; Published: November 06, 2015

Citation: Safdar OY, Shalaby M, Toffaha WM, Turki AA, Toffaha WM, et al., (2015) Neutrophil Gelatinase-Associated Lipocalin as an Early Marker for the Diagnosis of Urinary Tract Infections in Saudi Children. J Nephrol Ther 5: 221. doi:10.4172/21610959.1000221

Copyright: @ 2015 Safdar OY, et al. This is an open-access article distributed under the terms of the Creative Commons Attribution License, which permits unrestricted use, distribution, and reproduction in any medium, provided the original author and source are credited. 
AKI with high sensitivity 1-2 days before the serum creatinine levels increases $[9,10]$. Current studies have shown the worth of NGAL in identifying patients with AKI [11], i.e. it differentiates between acute pyelonephritis and lower UTI [12], and diagnoses UTI in adults in ICU settings [13].

Therefore, NGAL can be considered as a good marker for renal function and inflammation, providing more specificity for the diagnosis of AKI. Researches on urinary NGAL are limited in Saudi Arabia. It is believed that measuring urinary NGAL can be an effective screening tool to diagnose various renal diseases. In this study, we need to authorize the efficiency of computing urinary NGAL as a tool to diagnose UTI, with the hopes that medical therapy can be commenced early and potential complications can be avoided.

\section{Methods and Data Collection}

A cross-sectional study design was used to assess specificity and sensitivity of urinary NGAL in the early UTI diagnosis. The researcher recruited 73 patients ( 33 male and 40 female patients). The inclusion criteria included febrile children with an average temperature of $>38^{\circ} \mathrm{C}$ in the emergency or pediatric ward (0-14 years), who were clinically assessed by a physician and suspected to have UTI. The duration of the study was three months i.e. from June-August 2014.

The data was collected on data sheets including demographics (gender, date of birth, and race), chronic illness, previous documented UTI, presenting symptoms, vital signs, weight, blood glucose, a history of prophylactic Abs, type of antibiotics used, dipstick results, laboratory workup blood tests (including C-reactive protein (CRP), hemoglobin (HGB), white blood cells (WBCs), platelets, creatinine, blood culture, sensitivity (C\&S), and urine tests(including WBCs, red blood cells (RBCs), leukocytes, and urine C\&S). A UTI was diagnosed when the patients were presented with pyuria $(\geq 5 \mathrm{WBCs} / \mathrm{HPF})$ and positive urine culture(pure growth of $10^{5}$ organism $/ \mathrm{ml}$ ).

Urine samples for culture and NGAL analysis were obtained by catheter in infants and by midstream urine in older age groups. The correlation between NGAL and CRP levels were also examined.

\section{Ethical declaration}

The ethical approval for this study was granted by the ethics committee of King Abdulaziz University Hospital regarding studies in human subjects. Moreover, the study was approved by the ethics committee and the hospital administration. Informed and a written consent was acquired from the patients' parents before participating in the study.

\section{Statistical analysis}

Shapiro-Wilk's test was used to determine the distribution of continuous parameters (such as abnormal vs. normal). Based on the distribution of data, non-parametric tests were utilized to evaluate non-stop parameters among groups. Non-parametric Mann-Whitney $\mathrm{U}$ test was employed to examine the urinary NGAL levels and mean serum CRP with positive culture of urine in comparison to the negative cultures. A Spearman correlation was undertaken to examine if any relationship exists between urinary protein, urine NGAL, and estimated creatinine clearance. Analysis through ROC (Receiver operating curve) was used to examine the specificity and sensitivity of different cutoff points for uNGAL was used to predict UTI. The most suitable cutoff value that maximizes both sensitivity and specificity has been selected for both uNGAL and serum CRP. Hanley method is used to compare ROC analysis for UNGAL and serum CRP (14). The level of statistical significance was examined at $\mathrm{p}<0.05$.

\section{Results}

Out of 73 patients, 31 patients were suffering from UTIs, whereas 42 did not have UTIs. There were around 33 male and 40 female patients. The mean age of the patients with UTIs was 25 months, and the mean age of patients without UTIs was 27.3 months. The median urinary NGAL level in patients with UTIs was $56 \mathrm{ng} / \mathrm{ml}$, with an interquartile range of (29.1-82.9) $\mathrm{ng} / \mathrm{ml}$, whereas the median in patients without UTIs was $24 \mathrm{ng} / \mathrm{ml}$, with an interquartile range of (7.1-40.9) $\mathrm{ng} / \mathrm{ml}$. The median serum CRP level in patients with UTIs was $10 \mathrm{mg} / \mathrm{l}$, with an interquartile range of $(4.7-15.3) \mathrm{mg} / \mathrm{l}$, whereas the median inpatients without UTIs was7.2 $\mathrm{mg} / \mathrm{l}$, with an interquartile range of (3.3-11.2) $\mathrm{mg} / \mathrm{l}$.

\section{Comparison of urinary NGAL levels in patients with and without UTIs}

As the data were not normally distributed, the Mann-Whitney U test was used to compare the mean urinary NGAL levels. The mean rank of urinary NGAL was $43.7 \mathrm{ng} / \mathrm{ml}$ in patients with UTIs compared with $31.7 \mathrm{ng} / \mathrm{ml}$ in patients with negative culture results (Figure 1). Hence, with a p-value of 0.17 , the mean difference was non-significant.

For serum CRP levels, the mean rank was $38.5 \mathrm{mg} / \mathrm{l}$ in patients with UTIs in comparison to $35.8 \mathrm{mg} / \mathrm{l}$ in patients with negative culture results; therefore, the difference was not significant with $\mathrm{p}$-value 0.3 .

\section{Spearman correlation test}

According to Spearman correlation test, a significant correlation exists between NGAL and CRP levels (Table 1). This test is a statistical measure to determine the strength of a monotonic association between the selected variables. The more closer a value is to 1 , the stronger will be the relationship. The significant spearman correlation co-efficient value of 0.503 confirms, what is apparent in the Scatter plot in appendix that there appears to be a positive correlation between NGAL and CRP levels. Thus, large values of NGAL are associated with large CRP levels ( $\mathrm{rs}=0.503 \mathrm{n}=73, \mathrm{p}<.001$ ) (Figure 2).

\section{Roc curve analysis}

According to ROC curve analysis for NGAL levels, the area under the curve was 0.664 . The optimal cut-off value was $35.83 \mathrm{ng} / \mathrm{ml}$. The sensitivity was 0.594 , and the specificity was 0.683 . For serum CRP, the area under curve was 0.536 . The optimal cut-off value was calculated to be $6.9 \mathrm{mg} / \mathrm{l}$. The sensitivity is 0.53 and specificity is 0.47 (Figure 3). By using Hanley method, difference of the area under curve is 0.128. Moreover, this difference is not significant with p-value of 0.17 .

\section{Discussion}

To best of our knowledge, this is the first study in Saudi Arabia to examine the ability of urinary NGAL levels to act as a diagnostic biomarker for pediatric UTI. In this cross-sectional study, the urinary NGAL level was a poor marker for the early diagnosis of UTIs. These results contradict the findings of other studies conducted in both rats and humans, where urinary and serum NGAL levels served as a good marker for the early diagnosis of UTIs.

In mouse models, urinary NGAL microRNA and protein levels increased immediately after injecting bacteria into the kidney. The increase in urinary NGAL levels persists until 6 weeks post-injection [14]. In contrast to the results of our study, Sim et al. showed that urinary NGAL is an excellent marker for UTI diagnosis in a study 


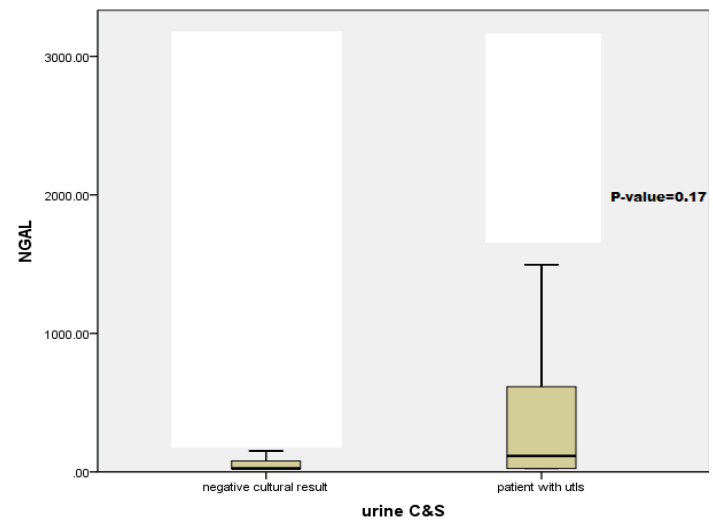

Figure 1: The mean urinary NGAL level is higher in patients with positive UTI versus negative UTI, the difference was not significant with p-value 0.17 .

\begin{tabular}{|c|c|c|c|c|}
\hline \multicolumn{5}{|l|}{ Correlations } \\
\hline & & & NGAL & CRP \\
\hline \multirow{6}{*}{ Spearman's rho } & \multirow{3}{*}{ NGAL } & Correlation Coefficient & 1.000 & $.503^{* *}$ \\
\hline & & Sig. (2-tailed) & . & .000 \\
\hline & & $\mathrm{N}$ & 80 & 80 \\
\hline & \multirow{3}{*}{ CRP } & Correlation Coefficient & $.503^{* *}$ & 1.000 \\
\hline & & Sig. (2-tailed) & .000 & . \\
\hline & & $\mathrm{N}$ & 80 & 80 \\
\hline
\end{tabular}

"Correlation is significant at the 0.01 level (2-tailed)

Table 1: Spearman's correlation test.

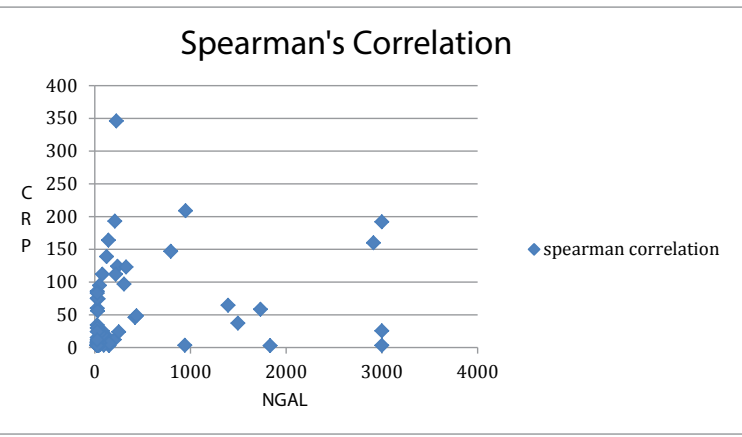

Figure 2: Spearman's correlation test result.

of 123 children. Urinary NGAL was a sensitive biomarker that was capable of distinguishing upper from lower UTI. The best predictive urinary cut-off value was $102 \mathrm{ng} / \mathrm{ml}$ [15]. The aforementioned study also demonstrated that urinary NGAL/creatinine and serum cystatin $\mathrm{C}$ levels can distinguish between upper and lower UTIs. Moreover, urinary NGAL/creatinine and KIM-1 levels can also distinguish cases of UTI (upper and lower) from the control group [16].

Unfortunately, in this study, we did not perform $99 \mathrm{mTc}$ dimercaptosuccinic acid (DMSA) scans, which would help to detect whether urinary NGAL could distinguish upper UTIs from lower UTIs. Another pediatric study in Turkey showed that urinary NGAL levels and the urinary NGAL/creatinine ratio were both predictive of UTI.

The optimal cut-off value in the Turkish study was $20 \mathrm{ng} / \mathrm{ml}$, while the optimum cut-off value in our study was $35.8 \mathrm{ng} / \mathrm{ml}$ [17]. Based on

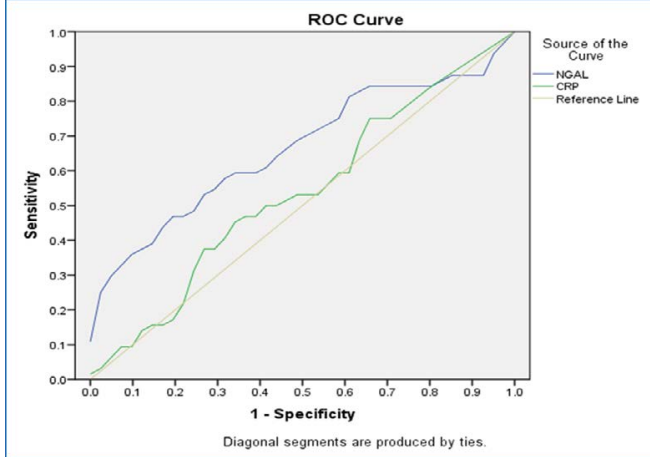

Figure 3: ROC curve analysis: for NGAL levels, the area under the curve was 0.664 . The optimal cut-off value was $35.83 \mathrm{ng} / \mathrm{ml}$. The sensitivity was 0.594 , and the specificity was 0.683 . For serum CRP, the area under the curve was 0.528 . The optimal cut-off value was $6.9 \mathrm{mg} / \mathrm{l}$. The sensitivity was 0.53 and the specificity was 0.47 .

these studies, it can be concluded that the measurement of urinary NGAL levels represents a rapid screening test for UTI diagnosis, helping to predict upper UTI versus lower UTI. This assist in determining appropriate duration of antibiotic treatment (pyelonephritis requires a longer course of antibiotics than isolated cystitis). However, a wide variety of optimal cut-off values for UTI diagnosis have been found among different clinical studies.

In this study, the author found a clear correlation between urinary NGAL and CRP levels. This finding is consistent with the results of other studies, indicating that NGAL can act as an acute phase reactant during an inflammatory process. This finding may not be surprising given that NGAL is released from neutrophils. Urinary NGAL can also serve as a good biomarker for monitoring the response to therapy. Recurrent febrile UTIs are related to the presence of vesicoureteral reflux and the development of renal scars [18]. To assess the effectiveness of urinary biomarkers in renal scar screening, Kitalo et al. measured the following biomarkers in the urine of 49 febrile infants: "NGAL, beta-2 microglobulin" (BMG), "liver-type fatty acid binding protein" (L-FABP), "N-acetyl- $\beta$-D-glucosaminidase" (NAG), as well as angiotensinogen (AGT). Only urinary angiotensinogen levels were positively correlated with the development of renal scars in febrile UTI [19]. However, another study demonstrated that urinary NGAL correlated with the development of renal scarring in 34 children with vesicoureteral reflux. Interestingly, limited association was found between the levels of urinary NGAL and reflux grade [20]

NGAL can also help to detect bacterial infection. NGAL is markedly augmented during bacterial infections in comparison to the viral infections, suggesting that NGAL can be precious diagnostic marker for distinguishing between viral and acute bacterial infections [15]. NGAL biosynthesis was known to be stimulated by bacterial overgrowth because NGAL has a bacteriostatic effect by depleting siderophores. The role of urinary and serum NGAL is not limited to UTI; NGAL is also related to acute tubular injury. The possible usefulness of NGAL as an AKI biomarker was documented because NGAL is considered to be most basic and rapidly induced genes in the kidney after nephrotoxic or ischemic injury in animal models [7]. In a study involving 143 children with septic shock, Derek et al. found that urinary NGAL levels are considered to be exceedingly predictive of the AKI developments. The median level of NGAL was $355 \mathrm{ng} / \mathrm{ml}$ in patients with AKI, in comparison to " $186 \mathrm{ng} / \mathrm{ml}$ " in patients without AKI [21]. Urinary NGAL levels can also help to distinguish pre-renal 
failure from intrinsic kidney disease. A study from Germany involved 87 subjects, including 24 patients with pre-renal AKI, 38 patients with intrinsic kidney disease, and 25 healthy subjects. The mean urinary NGAL level was $458 \mathrm{ng} / \mathrm{ml}$ in patients with intrinsic AKI compared with $64.8 \mathrm{ng} / \mathrm{ml}$ in patients with pre-renal AKI. This finding is clinically important because the management of pre-renal AKI differs from that of intrinsic AKI [22].

NGAL has also been studied in other kidney diseases, including autosomal polycystic kidney disease [23] and systemic lupus nephritis [24]. It is unclear why urinary NGAL did not perform well as a diagnostic marker for UTI in our study. There are several limiting factors in our study. This study only recruited a small number of patients and was sampled from a single center. Additionally, the study did not perform a DMSA scan, which is considered an excellent imaging tool to diagnose upper UTI. It would have permitted the author to test the efficacy of urinary NGAL for distinguishing upper from lower UTI. We found both uNGAL and serum CRP as poor markers for UTI diagnosis. We used ROC analysis, which is a good indicator for examining sensitivity and specificity but it has limitations as it relies on assumption of the best threshold point for clinical performance and is subjected to bias. The patients were not followed up regarding the presence of vesicoureteral reflux and renal scarring, which is known to be associated with the development of hypertension, and chronic kidney disease. We believe that a multicenter study with a larger sample size will assist to evaluate the role of urinary NGAL as an early diagnostic measure for UTI. Moreover, more follow up time is needed to assess the possible role of urinary NGAL as a predictive test for long-term sequelae from UTI such as renal scarring, hypertension, and impaired renal function.

\section{Conclusions}

Urinary NGAL is not an adequate predictor for the early diagnosis of UTI in children. However, it is recommended that multicenter studies including a larger number of patients should be conducted to confirm these findings. The relationship of urinary NGAL with the occurrence of vesicoureteral reflux and renal scars development must be assessed in a prospective manner.

\section{Conflict of Interest}

None of the authors of this manuscript have any conflicts of interest.

\section{Acknowledgments}

The authors thank all of the participants and volunteers, who took part in the study with their immense support and help.

\section{References}

1. Kliegman RM, Behrman RE, Jenson HB, Stanton BF (2007) Nelson text book of pediatrics, (18 $18^{\text {th }}$ edtn), Saunders, Philadelphia.

2. White $B$ (2011) Diagnosis and treatment of urinary tract infections in children. Am Fam Physician 83: 409-415.

3. Montini G, Tullus K, Hewitt IK (2011) Febrile urinary tract infections in children. N Engl J Med 365: 239-250.

4. Han WK, Bonventre JV (2004) Biologic markers for the early detection of acute kidney injury. Curr Opin Crit Care 10: 476-482.

5. Kjeldsen L, Bainton DF, Sengelov H, Borregaard N (1993) Structural and functional heterogeneity among peroxidase-negative granules in human neutrophils: identification of a distinct gelatinase-containing granule subset by combined immunocytochemistry and subcellular fractionation. Blood 82: 31833191.

6. Devarajan P (2010) Review: neutrophil gelatinase-associated lipocalin: a troponin-like biomarker for human acute kidney injury. Nephrology (Carlton) 15 419-428.
7. Mishra J, Dent C, Tarabishi R, Mitsnefes MM, Ma Q, et al. (2005) Neutrophilgelatinase-associated lipocalin (NGAL) as a biomarker for acute renal injury after cardiacsurgery. Lancet 365 : 1231-1238.

8. Devarajan P (2008) Neutrophil gelatinase-associated lipocalin-an emerging troponin for kidney injury. Nephrol Dial Transplant 23: 3737-3743.

9. Al-Ismaili Z, Palijan A, Zappitelli M (2011) Biomarkers of acute kidney injury in children: discovery, evaluation, and clinical application. Pediatr Nephrol 26: 29-40.

10. Dent CL, Ma Q, Dastrala S, Bennett M, Mitsnefes MM, et al. (2007) Plasma neutrophil gelatinase-associated lipocalin predicts acute kidney injury, morbidity and mortality after pediatric cardiac surgery: a prospective uncontrolled cohort study. Crit Care 11: R127

11. Antonucci E, Lippi G, Ticinesi A, Pigna F, Guida L, et al. (2014) Neutrophil gelatinase-associated lipocalin (NGAL): a promising biomarker for the early diagnosis of acute kidney injury (AKI). Acta Biomed 85: 289-294.

12. Seo WH, Nam SW, Lee EH, Je BK, Yim HE, et al. (2014) A rapid plasma neutrophil gelatinase-associated lipocalin assay for diagnosis of acute pyelonephritis in infants with acute febrile urinary tract infections: a preliminary study. Eur J Pediatr 173: 229-232.

13. de Geus HR, Bakker J, Lesaffre EM, le Noble JL (2011) Neutrophil gelatinaseassociated lipocalin at ICU admission predicts for acute kidney injury in adult patients. Am J Respir Crit Care Med 183: 907-914.

14. Ichino M, Kuroyanagi Y, Kusaka M, Mori T, Ishikawa K, et al. (2009) Increased urinary neutrophil gelatinase associated lipocalin levels in a rat model of upper urinary tract infection. J Urol 181: 2326-2231.

15. Sim JH, Yim HE, Choi BM, Lee JH, Yoo KH (2015) Plasma neutrophil gelatinaseassociated lipocalin predicts acute pyelonephritis in children with urinary tract. Pediatr Res 78: 48-55.

16. Yim HE, Yim H, Bae ES, Woo SU, Yoo KH (2014) Predictive value of urinary and serum biomarkers in young children with febrile urinary tract infections. Pediatr Nephrol 29: 2181-2189.

17. Yilmaz A, Sevketoglu E, Gedikbasi A, Karyagar S, Kiyak A, et al. (2009) Early prediction of urinary tract infection with urinary neutrophil gelatinase associated lipocalin. Pediatr Nephrol 24: 2387-2392.

18. Leroy S, Fernandez-Lopez A, Nikfar R, Romanello C, Bouissou F, et al. (2013) Association of procalcitonin with acute pyelonephritis and renal scars in pediatric UTI. Pediatrics 131: 870-879.

19. Kitao T, Kimata T, Yamanouchi S, Kato S, Tsuji S, et al. (2015) Urinary biomarkers for screening of renal scar in children with febrile urinary tract infection. J Urol 194: 766-771

20. Ichino M, Kusaka M, Kuroyanagi Y, Mori T, Morooka M, et al. (2010) Urinary neutrophil-gelatinase associated lipocalin is a potential noninvasive marker for renal scarring in patients with vesicoureteral reflux. J Urol 183: 2001-2007.

21. Wheeler DS, Devarajan P, Ma Q, Harmon K, Monaco M, et al. (2008) Serum neutrophil gelatinase-associated lipocalin (NGAL) as a marker of acute kidney injury in critically ill children with septic shock. Crit Care Med 36: 1297-1303.

22. Seibert FS, Pagonas N, Arndt R, Heller F, Dragun D, et al. (2013) Calprotectin and neutrophil gelatinase-associated lipocalin in the differentiation of pre-renal and intrinsic acute kidney injury. Acta Physiol (Oxf) 207: 700-708.

23. Bolignano D, Coppolino G, Campo S, Aloisi C, Nicocia G, et al. (2007) Neutrophil gelatinase-associated lipocalin in patients with autosomal-dominant polycystic kidney disease. Am J Nephrol 27: 373-378.

24. Brunner HI, Mueller M, Rutherford C, Passo MH, Witte D, et al. (2006) Urinary neutrophil gelatinase-associated lipocalin as a biomarker of nephritis in childhood-onset systemic lupus erythematosus. Arthritis Rheum 54: 2577 2584 